tional photography. The effective exposure times with the converter and motion picture camera were $\mathrm{I} / 2$ oth second or less. Since the dark emission of the converter was unimportant for such short exposures, the operating potential could be increased enough to yield speed gains of about Ioo-fold.

The cascaded converter is an extremely simple device to use. It requires no specially prepared plates, no home-made photocathodes, no external vacuum system, and no liquid-air traps. It requires only a voltage supply and a fast camera lens.

This work is being carried out under the Carnegie Institution with the support of the National Science Foundation.

$$
\begin{array}{r}
\text { Carnegie Institution of Washington } \\
\text { Washington, D. C., } \\
\text { Lowell Observatory } \\
\text { Flagstaff, Ariz. and } \\
\text { Mt. Wilson and Palomar Observatories } \\
\text { Pasadena, California }
\end{array}
$$

Underhill, Anne B. The hydrogen lines of 9 Sagittae, O7f.

Very little quantitative data on the shapes and strengths of lines in O-type spectra exist. This paper presents equivalent widths and line profiles of the Balmer lines of hydrogen in the spectrum of 9 Sagittae, O7f. The observations were obtained with the 72 -inch telescope and cassegrain spectrograph during the years $195^{\circ}$ to 1958 . The resolution is sufficient that the apparent profiles require no correction for instrumental effects. No secular changes in this spectrum were observed except in the emission feature at $H \alpha$.

The $H \alpha$ emission is variable in intensity being stronger in 1957 than in I95I or I953, the only years in which observations of this line were obtained. The total extent of the emission is $\pm \mathrm{I} 2 \AA$, corresponding to a velocity displacement of $\pm 540 \mathrm{~km} / \mathrm{sec}$. The emission is due to hydrogen only since the $4 n$ series of $H e$ II which blends with the Balmer lines to about $H_{\mathrm{IO}}$ is entirely in absorption. Any broad absorption feature due to $H e$ II and $H$ that might exist at $H \alpha$ is entirely filled in with emission.

The absorption profile at $H \beta$ has a somewhat peculiar, shallow shape. It is interpreted as a broad strong absorption due to HeII and $H$ which has partially been filled in with emission from hydrogen.

The profiles of the lines $H_{\gamma}$ to $H_{1} 6$ are entirely in absorption. The HeII lines blend on the violet side causing an unsymmetric profile. The Balmer series terminates at $H_{1} 6$ which is clearly present as a moderately strong line blended with OIII and $H e r$. There is no line of comparable strength near the position of $\mathrm{H}_{\mathbf{I}} 7$ or of any higher member of the Balmer series. This truncation of the series indicates that Stark effect is the major broadening agent of the hydrogen lines. A comparison of the apparent wings of $H_{\text {Io }}$ to $H_{\text {I } 4}$ with those of $H \gamma, H \delta$, and $H \epsilon$ leads to the conclusion that the apparent continuum at $\lambda$ $<3900 \AA$ is depressed by overlapping of the wings of the hydrogen lines even though the ultra-violet hydrogen lines appear to be well separated. According to the Teller-Inglis formula the electron density is $2 \times \mathrm{IO}^{14}$.

\section{Dominion Astrophysical Observatory} Victoria, B. C., Canada

\section{van den Bergh, S. The luminosity classification of galaxies.}

The degree to which spiral structure is developed in galaxies is strongly correlated with absolute luminosity. This correlation is in the sense that the brightest galaxies have the most highly developed spiral arms.

A two-parameter classification system has been developed which assigns a type and a luminosity class to each galaxy. By means of this classification system the absolute magnitudes of $\mathrm{Sb}, \mathrm{Sc}$ and Ir galaxies may be estimated with an accuracy of about half a magnitude on the prints of the Palomar Sky Survey.

The appearance of many spiral galaxies which are members of physical pairs or dense clusters differs from that of isolated spirals of the same type and luminosity. Sb galaxies which are members of pairs or clusters often have fuzzier spiral arms than isolated galaxies of the same type. The appearance of such galaxies on the Sky Survey blue prints is quite similar to the appearance of isolated $\mathrm{Sb}$ galaxies on the red prints. This suggests that the luminosity function of star creation may be deficient in bright OB stars in many $\mathrm{Sb}$ galaxies which are members of close pairs or dense clusters. Sc spirals in clusters tend to have patchier spiral arms than isolated Sc galaxies.

The differences between isolated and cluster galaxies may be due to the after-effects of tidal interactions. There appears to be a distinct possibility that such galaxies which have suffered 